$\xi=-$ 国

\title{
Caputo-Fabrizio Time Fractional Derivative Applied to Visco Elastic MHD Fluid Flow in the Porous Medium
}

\author{
Salah Uddin ${ }^{1 *}$, M. Mohamad ${ }^{1}$, M. A. H. Mohamad ${ }^{4}$, Suliadi Sufahani ${ }^{1}$, MGhazali Kamardan ${ }^{1}$, Obaid Ullah

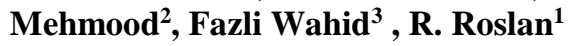 \\ ${ }^{I}$ Department of Mathematics and Statistics, Faculty of Applied Sciences and Technology, Universiti Tun Hussein Onn Malaysia, Pagoh \\ Educational Hub,84600 Pagoh, Johor, Malaysia \\ ${ }^{2}$ Faculty of Science, Deprartment of Mathematics, Comsats Institute of Information Technology, Wah Cantt, 47040, Pakistan \\ ${ }^{3}$ Faculty of computer science and Information Technology, Universiti Tun Hussein Onn Malaysia, , 86400 Batu Pahat, Johor, Malaysia \\ ${ }^{4}$ Faculty of Diploma Study Centre, Department of Mechanical Engineering, Universiti Tun Hussein Onn Malaysia, Pagoh Educational \\ Hub,84600 Pagoh, Johor, Malaysia \\ *Corresponding author E-mail: salahuddinjalil@gmail.com
}

\begin{abstract}
In this paper the laminar fluid flow in the axially symmetric porous cylindrical channel subjected to the magnetic field was studied. Fluid model was non-Newtonian and visco elastic. The effects of magnetic field and pressure gradient on the fluid velocity were studied by using a new trend of fractional derivative without singular kernel. The governing equations consisted of fractional partial differential equations based on the Caputo-Fabrizio new time-fractional derivatives $\mathrm{NFD}_{t}$. Velocity profiles for various fractional parameter a, Hartmann number, permeability parameter and elasticity were reported. The fluid velocity inside the cylindrical artery decreased with respect to Hartmann number, permeability parameter and elasticity. The results obtained from the fractional derivative model are significantly different from those of the ordinary model.
\end{abstract}

Keywords: Laminar flow, Porosity, Hartmann number.

\section{Introduction}

MHD fluid flow simulated from the local and non-local models and its parameter due to wall elasticity, wall permeability and pulsatile nature (due to external force) are gaining significant attention nowadays. The study of MHD fluid flow by using fractional derivatives is useful as it can provide many important scientific discoveries.

Gul et al [1] observed that Ec (Eckert number) affected the temperature profile and the flow velocity decreased with respect to $\omega$. Meanwhile, near the walls velocity was higher than that in the core region. Raza [2] determine the behaviors of velocity and shear stress were similar in the $2 \mathrm{D}$ rotational flow problem where the working fluid, i.e., the second grade fluid was modeled by using the fractional Caputo-time derivative. Abro et al [3] and [4] investigated the effects of Caputo-Fabrizio fractional parameter, material parameter, rheological parameter and nondimensional parameter on fluid flow. Both slip and no-slip boundary conditions were considered in a permeable medium by using a newly defined M-function. They reported that Newtonian fluid was the slowest. According to Sharma and Gupta [5] the rate of transport decreased when the magnetic field was considered. A complex generalized differential quadrature method employing the fourthorder Runge-Kutta method was used [6] to solve the nonlinearity problem. A 3D flow model was transformed into 2D MHD Jeffery Hamel flow model [7], which was further converted into an equivalent non-linear model described by the third-order ordinary differential equation. The neural network models were developed by using $\log$ sigmoid activation function to the new transformed equations. It is able to solve other non-linear problems with singularities. Excellent agreement between the numerical results and analytical ones was reported [8]. During systoles and diastoles flow rotation was evident at the boundary. Meanwhile, the flow velocity was the maximum at the channel core region. In [9], flow in stenosed inclined tubes with periodic body acceleration was simulated and it was found that both velocity and volumetric flow rate decreased. For a particular range of phase angle, wall shear stress increased by as Hartmann number increased (for various cardiovascular diseases). The exact solutions for MHD flow under periodic body acceleration were derived in [10]. The method was more precise than other numerical methods. In [11], generalizedBurgers' equation was solved and the velocity was smaller in the absence of magnetic field. The effects of external magnetic field and porosity on the blood flow inside the channel were highlighted by [12]. The outcomes are helpful in understanding the blood circulation in human body. The flow models employing sumudu and finite Hankel transform which were used to simulate viscous MHD flow in a circular cylinder [13] were very attractive numerically. Fractional calculus approach was used to solve an incompressible generalized Oldroyd-B fluid [14]. The solutions were expressed in terms of Fox H-function by using Laplace transformation. The temperature of MHD flow subjected to thermal radiation and exponentially porous stretching sheet decreased as the stratified parameter increased. Following Mohan et al [12], the new definition of fractional derivative without singular kernel was used to model fluid flow (subjected to magnetic field) inside an artery. Good agreement has been found (see Figure (2)). In the current work, the effects of NFDt, magnetic field and other pa- 
rameters on fluid flow were studied analytically. Mathematica was used for simulation purpose.

\section{Mathematical modeling}

In this paper, an axially symmetric incompressible fluid flow model was developed. The flow was accelerated along the radius of the circular channel as shown in Figure 1. The pressure gradient acting along the channel is given as

$-\frac{\partial p}{\partial z}=A_{0}+A_{1} \cos (\omega t)$ for $t \geq 0$,

$G=a_{0} \cos \left(\omega_{1} t+\varphi\right)$

$A_{0}$ is the steady state part of the pressure gradient. $A_{1}$ is the amplitude of the oscillatory part, where $\omega=2 \pi f$, ( $f$ is the pulse frequency). $a_{0}$ is the amplitude of the body acceleration. Also $\omega_{1}=2 \pi f_{1}$, where $f_{1}$ is the body acceleration. Phase difference is represented as $\varphi$.
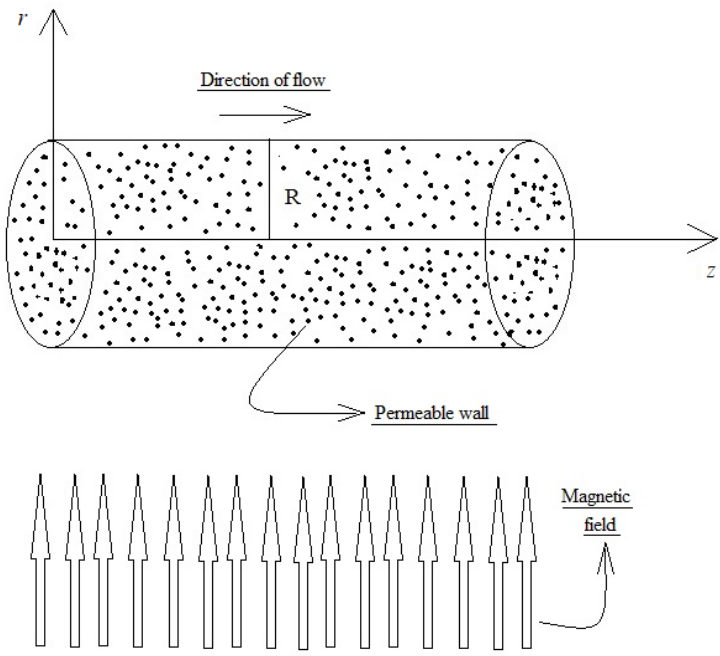

Fig. 1: Fluid flow geometry

$\rho \frac{\partial u}{\partial t}=A_{0}+A_{1} \cos (\omega t)+a_{0} \cos \left(\omega_{1} t+\varphi\right)+$

$\left(\mu+\mu_{1} \frac{\partial}{\partial t}\right)\left(\frac{\partial^{2} u}{\partial r^{2}}+\frac{1}{r} \frac{\partial u}{\partial r}\right)-k u-\sigma B_{0}{ }^{2} u$,

where $u(r, t)$ is the velocity component in $z$-direction, $\rho$ blood density, $\mu$ is viscosity, $\mu_{1}$ is the viscosity coefficient, $\sigma$ is electrical conductivity, $k$ is the permeability influence and $r$ is radial coordinate.

In the time fractional model, the governing equation discussed in (3) becomes

$$
\begin{aligned}
& \rho D_{t}^{(\alpha)} u=A_{0}+A_{1} \cos (\omega t)+a_{0} \cos \left(\omega_{1} t+\varphi\right)+ \\
& \left(\mu+\mu_{1} \frac{\partial}{\partial t}\right)\left(\frac{\partial^{2} u}{\partial r^{2}}+\frac{1}{r} \frac{\partial u}{\partial r}\right)-k u-\sigma B_{0}{ }^{2} u,
\end{aligned}
$$

where
$D_{t}^{(\alpha)} f(t)=\frac{M(\alpha)}{1-\alpha} \int_{a}^{t} \dot{f}(\tau) e^{-\frac{\alpha(t-\tau)}{1-\alpha} d \tau}$,

with $\alpha \in[0,1], a \in[-\infty, t)$ and $f \in L^{1}(a, b), b>a$, where $L^{1}(a, b)$ is the class of all integrate able functions $f$ on $[a, b]$, $D_{t}^{(\alpha)}$ is called the new fractional time derivative $\mathrm{NFD}_{t}$.

Dimensionless and after dropping dashes, eq. (4) can be written as

$\alpha_{1}^{2} D_{t}^{(\alpha)} u=A_{0}+A_{1} \cos (t)+a_{0} \cos (b t+\varphi)+$

$\left(1+\beta \frac{\partial}{\partial t}\right)\left(\frac{\partial^{2} u}{\partial r^{2}}+\frac{1}{r} \frac{\partial u}{\partial r}\right)-\left(H^{2}+M^{2}\right) u$.

The dimensionless initial and boundary conditions are

$u(r, 0)=0, u(1, t)=0$, and $u(0, t)$ is finite

where $\alpha_{1}=R \sqrt{\frac{\omega \rho}{\mu}}$ is a Womersley parameter, $\beta=\frac{\omega \mu_{1}}{\mu}$ is the visco-elastic dimensionless parameter, $H=R \sqrt{\frac{k}{\mu}}$ is the porosity parameter, $M=R B_{0} \sqrt{\frac{\sigma}{\mu}}$ is the Hartmann number and $b=\frac{\omega}{\omega_{1}}$ is the radius tube.

\section{Solution to the problem}

By applying Laplace transform with respect to $t$ and finite Hankel transformation of order zero with respect to $r$ on (6) as well as using the boundary conditions stated in (7), we obtain

$\alpha_{1}^{2}\left(\frac{s \bar{u}_{H}\left(r_{n}, s\right)}{s+\alpha(1-s)}\right)=\left(\begin{array}{l}\frac{A_{0}}{s}+\frac{A_{1} s}{s^{2}+1}+ \\ \frac{a_{0}(s \cos \varphi-b \sin \varphi)}{s^{2}+b^{2}}\end{array}\right) \frac{J_{1}\left(r_{n}\right)}{r_{n}}-$

$r_{n}^{2}\left(\bar{u}_{H}\left(r_{n}, s\right)+\frac{\beta s \bar{u}_{H}\left(r_{n}, s\right)}{s+\alpha(1-s)}\right)-\left(H^{2}+M^{2}\right) \bar{u}_{H}\left(r_{n}, s\right)$,

where $\bar{u}_{H}\left(r_{n}, s\right)=\int_{0}^{1} r \bar{u}(r, s) J_{0}\left(r r_{n}\right)$ is the finite Hankel transform of the function $\bar{u}(r, s)$ and $r_{n}, n=1,2, \ldots$ being positive roots of Bessel function represented by $J_{0}(r)$, of first kind and zeroth order.

After performing inverse Hankel transformation and inverse Laplace transformation on the image function $\bar{u}_{H}\left(r_{n}, s\right)$ in (8) followed by using Robotnov/Hartley and Miller-Ross functions defined by,

$$
\begin{aligned}
& L^{-1}\left(\frac{1}{s^{q}-a}\right)=F_{q}[a, t]=\sum_{n=0}^{\infty} \frac{a^{n} t^{(n+1) q-1}}{\Gamma((n+1) q)}, \\
& L^{-1}\left(\frac{s^{-v}}{s-a}\right)=E_{t}(v, a)=\sum_{n=0}^{\infty} \frac{a^{k} t^{k+v}}{\Gamma(v+k+1)} .
\end{aligned}
$$




\subsection{Axial velocity in the Fractional model}

From (8) we have

$$
u(r, t)=2 \sum_{n=1}^{\infty} \frac{J_{0}\left(r r_{n}\right)}{r_{n} J_{1}\left(r_{n}\right)}\left\{\begin{array}{l}
h_{n}\left(e^{-c_{n} t}\left(1-\frac{f_{n}}{c_{n}}\right)+\frac{f_{n}}{c_{n}}\right)+ \\
j_{n} \cos t * m_{n}+k_{n} \cos (b t+\varphi)
\end{array}\right\}
$$

for $0<\alpha<1$

\subsection{Axial velocity in the Local model}

$u(r, t)=2 \sum_{n=1}^{\infty} \frac{J_{0}\left(r r_{n}\right)}{d_{n 1} r_{n} J_{1}\left(r_{n}\right)}\left\{\begin{array}{l}A_{0} \frac{1-e^{-c_{n 1} t}}{c_{n 1}}+ \\ A_{1} \cos t * e^{-c_{n 1} t}+ \\ a_{0} \cos (b t+\varphi) * e^{-c_{n 1} t}\end{array}\right\}$

for $\alpha=1$.

The blood velocities were simulated from the fractional fluid and ordinary fluid models expressed in (11) and (12), respectively. The parameters introduced in the axial velocity in order to reduce the step size were given as follows:

$$
\begin{aligned}
& a_{n}=\alpha_{1}^{2}+\beta r_{n}^{2}, b_{n}=H^{2}+M^{2}+r_{n}^{2}, c_{n}=\frac{b_{n} \alpha}{a_{n}+b_{n}-b_{n} \alpha}, \\
& d_{n}=a_{n}+b_{n}-b_{n} \alpha, e_{n}=1-\alpha, f_{n}=\frac{\alpha}{1-\alpha}, g_{n}=\frac{d_{n}}{e_{n}}, h_{n}=\frac{A_{0}}{g_{n}}, \\
& j_{n}=\frac{A_{1}}{g_{n}}, k_{n}=\frac{a_{0}}{g_{n}}, m_{n}=\left(f_{n}-c_{n}\right) e^{-c_{n} t}, d_{n 1}=\left.d_{n}\right|_{\alpha=1}=a_{n}, \\
& c_{n 1}=\left.c_{n}\right|_{\alpha=1}=\frac{b_{n}}{a_{n}} .
\end{aligned}
$$

Both fluid models were used to study the blood flow within the viscoelastic porous artery subjected to an externel magnetic field. In (11 and 12) $f * g$ represents the convolution product of $f$ and $g$.

\section{Graphical results and discussion}

Flow simulation was performed by using Mathematica software. The numerical results were presented in Figures (2-14). Both fractional and ordinary models were studied and the results were compared. The effects of Hartmann number, porosity parameter and elastic viscosity on fluid flow velocity were studied. In all of the cases few rational values of the fractional parameter less than 1 are compared with the ordinary model by setting $\alpha=1$. To evaluate the fluid flow velocity, the positive roots of the Bessel function $J_{0}(r)$ were calculated by using the coding method. We have considered the first 10 terms of the series while performing the inverse Hankel transform. The blood flow velocity was obtained by using Mathematica software. The curves of (11 and 12) were plotted against $r$ for different values of $M, H$ and $\beta$, and for fractional parameter $\alpha \in\{0.4,0.6,0.8,1.0\}$. The following constant values were used i.e., $a_{0}=3, A_{0}=2, A_{1}=4, \varphi=\pi / 12, t=0.5, b=1$ and $\alpha_{1}=1$.

Figures (3-6) show the influence of Hartmann number on the fluid flow velocity. The velocities predicted by using the two different models were somewhat different. It was noticed that, fluid flow velocity decreased as the strength of the external magnetic field increased. Figures (7-10) show the effect of porosity parameter on the fluid velocity. In order to examine the effect of porosity parameter on the fluid flow velocity, various values are assigned to $\mathrm{H}$. Fluid flow velocity decreased with respect to the porosity parameter. The effect of elastic viscosity on fluid flow velocity was shown in Figures (11-14). As the elastic viscosity increased, the fluid underwent larger resistance.

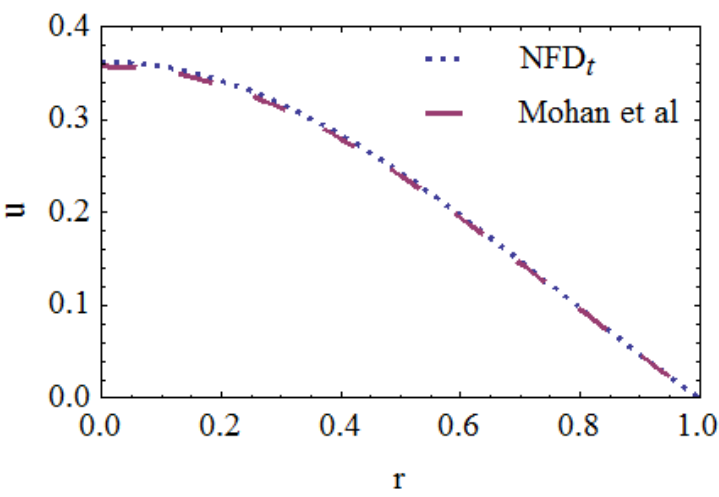

Fig: 2: Axial velocity calculated by Mohan et al and $\mathrm{NFD}_{t}$

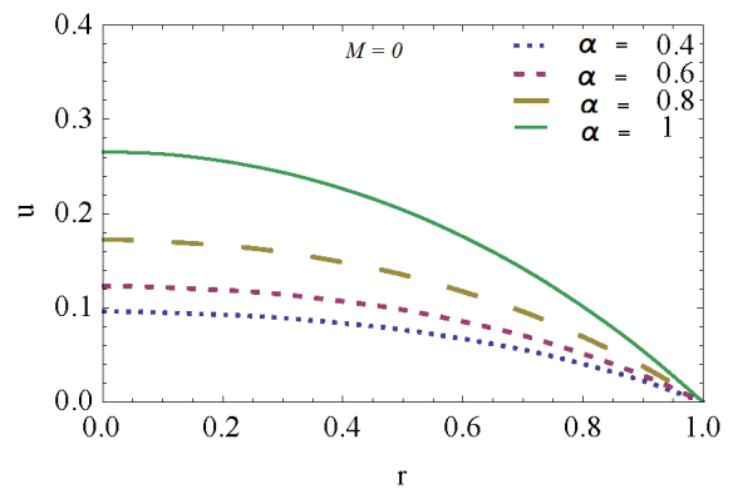

Fig.3: Axial velocity for various values of $\alpha$ and $M=0$ against $r$.

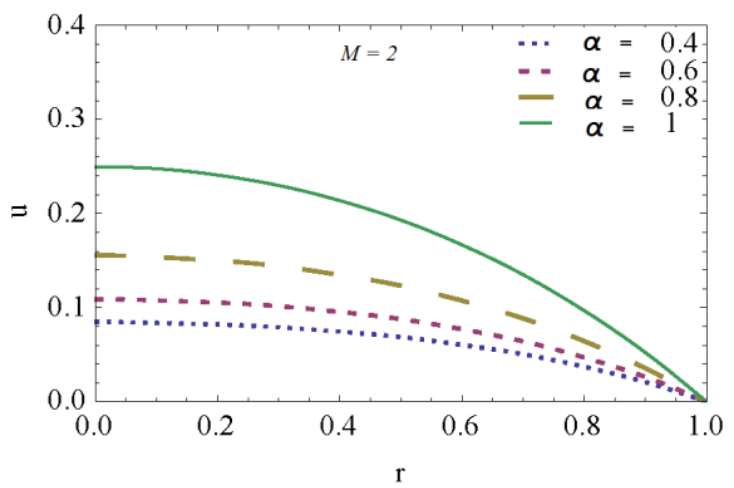

Fig.4: Axial velocity for various values of $\alpha$ and $M=2$ against $r$.

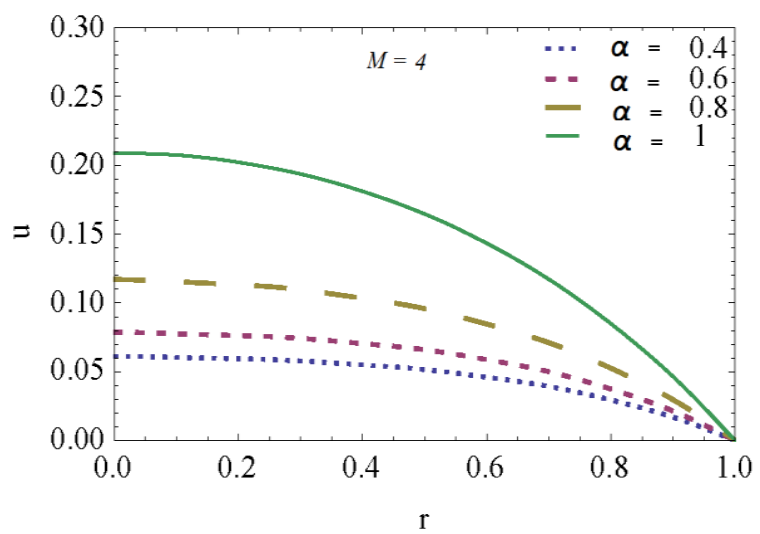

Fig.5: Axial velocity for different values of $\alpha$ and $M=4$ against $r$ 


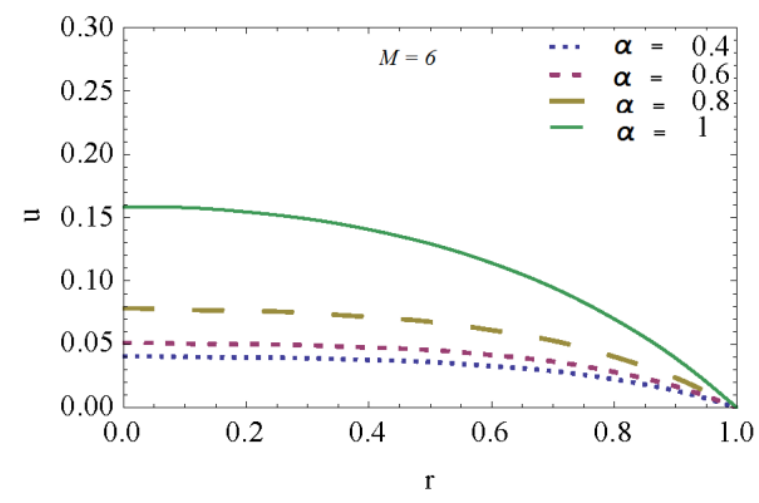

Fig.6: Axial velocity for various values of $\alpha$ and $M=6$ against $r$.

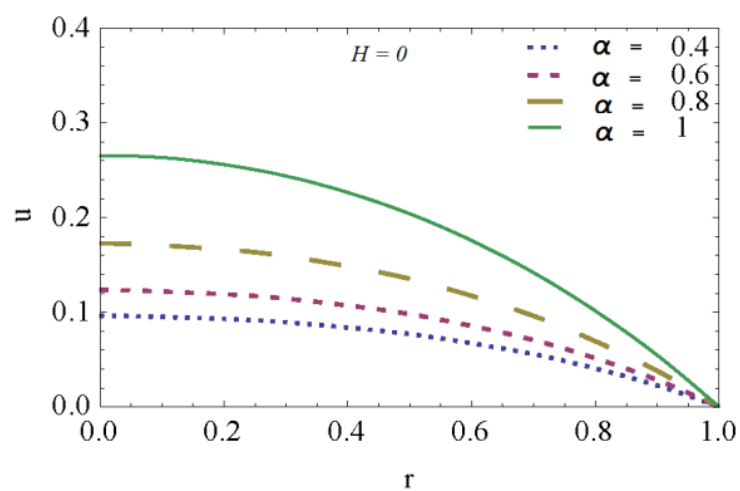

Fig.7: Axial velocity for various values of $\alpha$ and $H=0$ against $r$.

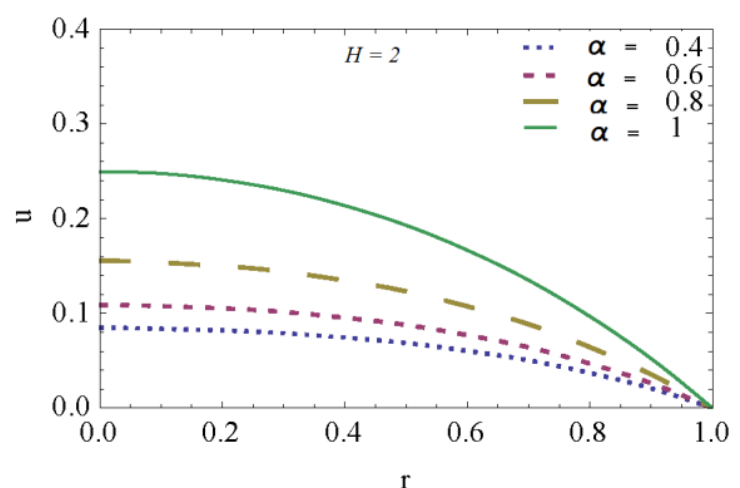

Fig.8: Axial velocity for various values of $\alpha$ and $H=2$ against $r$.

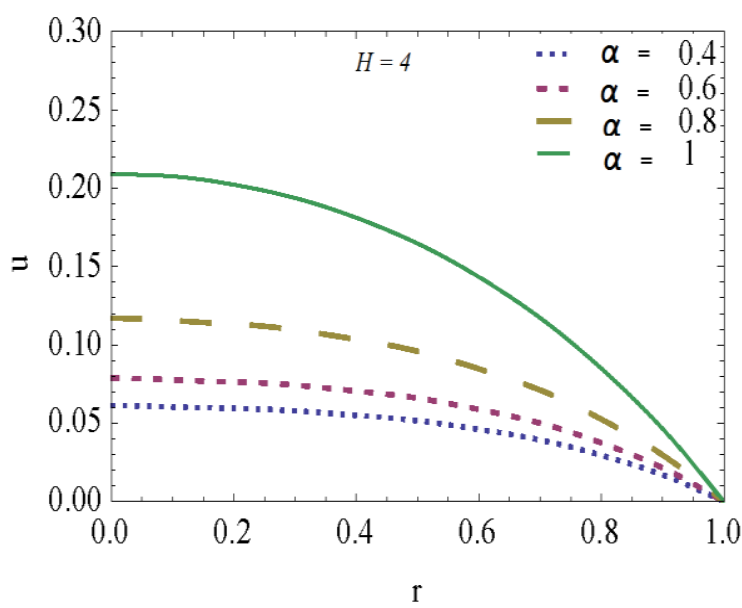

Fig.9: Axial velocity for various values of $\alpha$ and $H=4$ against $r$.

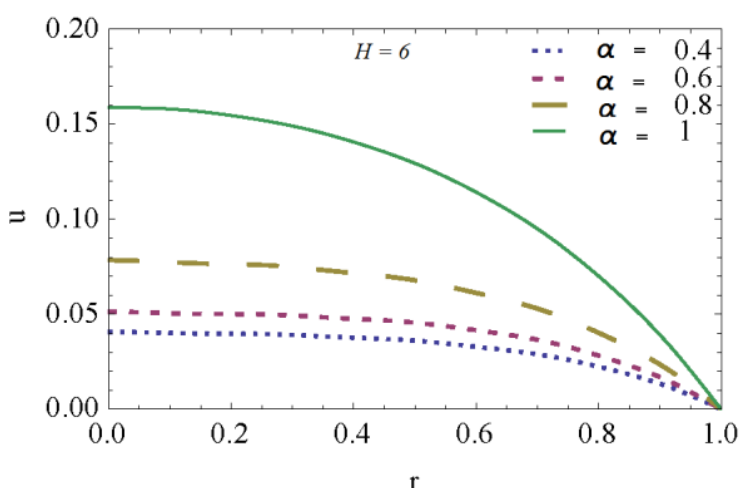

Fig.10: Axial velocity for various values of $\alpha$ and $H=6$ against $r$.

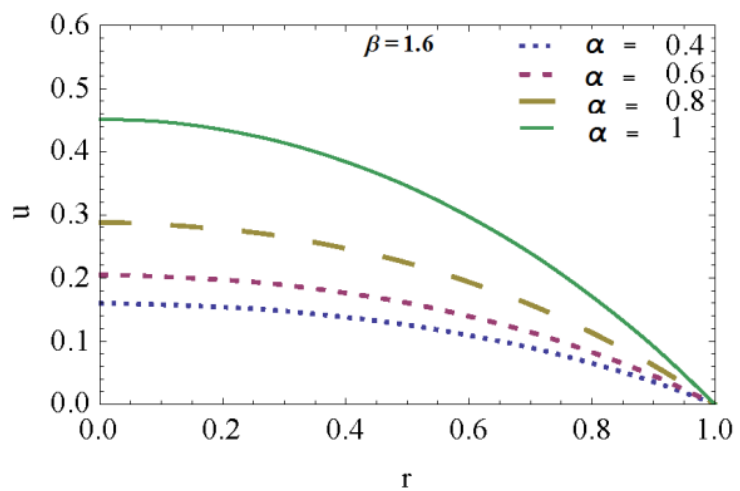

Fig.11: Axial velocity for various values of $\alpha$ and $\beta=1.6$ against $r$.

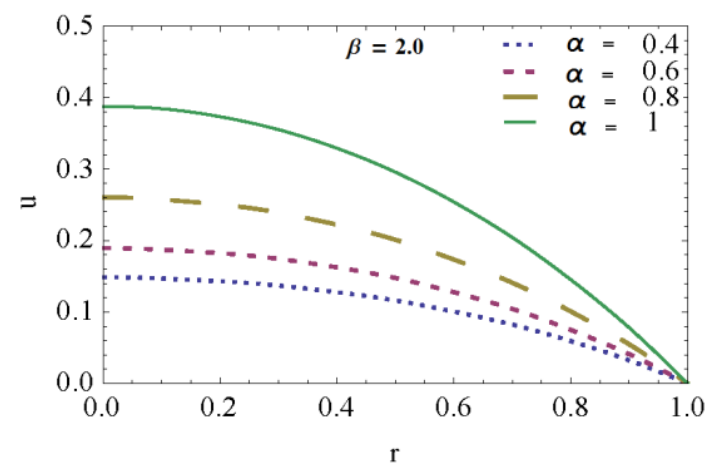

Fig.12: Axial velocity for various values of $\alpha$ and $\beta=2.0$ against $r$.

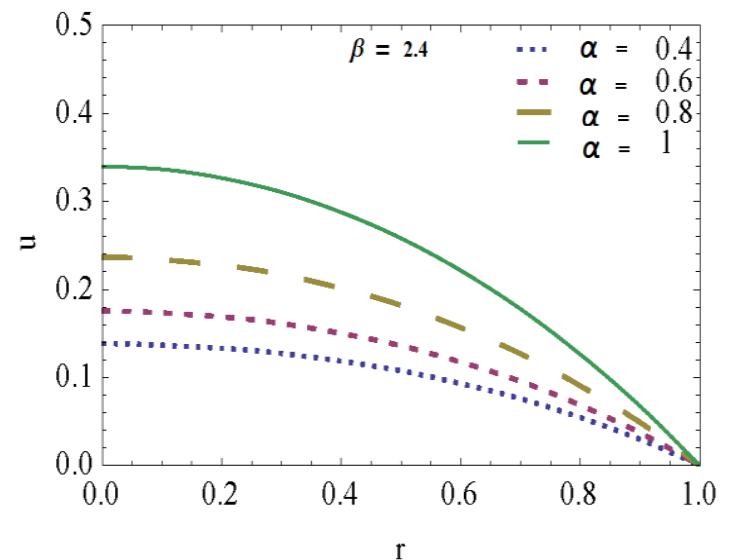

Fig.13: Axial velocity for different values of $\alpha$ and $\beta=2.4$ against $r$. 


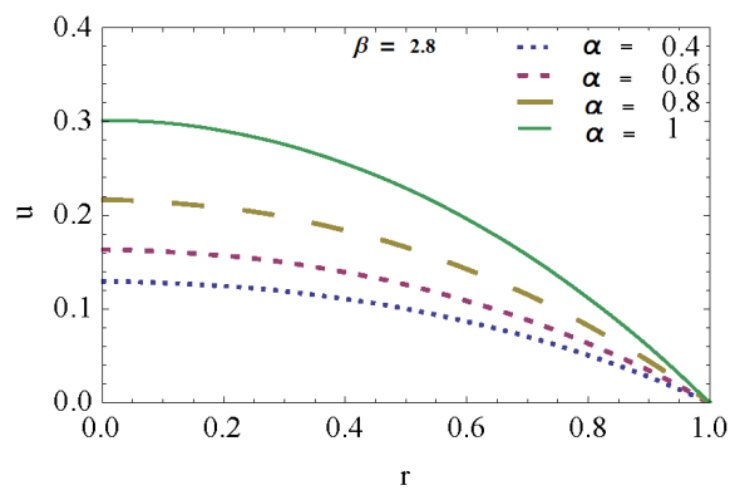

Fig.14: Axial velocity for various values of $\alpha$ and $\beta=2.8$ against $r$.

\section{Conclusion}

The effects of Caputo's new time fractional derivative $\mathrm{NFD}_{t}$, Hartmann number, porosity parameter and elasticity on the velocity of fluid flow subjected to magnetic field inside the cylindrical artery have been investigated. Both Laplace and finite Hankel transforms have been used to derive the analytical solutions of the governing equations containing non-linear fractional order derivative. The fluid velocity has been found to be inversely related to the Hartmann number and the porosity parameter in both fractional and ordinary models. Moreover in the case of an elastic viscosity for fractional model, the variation is very slow than as compared to the ordinary model. Therefore it is beneficial to study the flow behaviour using the non-local system as it contains much useful information on bio-fluid flow problems.

\section{Acknowledgment}

I would like to acknowledge the financial aid received from the Research, Innovation, Commercialization and Consultancy Management (ORICC) under Tier 1/H158 grant and Centre for Graduate Studies Universiti Tun Hussein Onn Malaysia.

\section{References}

[1] Gul T, Rehman I, Islam S, Khan MA, Ullah W \& Shah Z (2017), Unsteady third order fluid flow with heat transfer between two vertical oscillating plates, J. Appl. Environ. Biol. Sci, 5(4), pp.72-79.

[2] Raza N (2017), Unsteady rotational flow of a second grade fluid with non- integer Caputo time fractional derivative, Journal of Mathematics, 49(3), pp.15-25.

[3] Abro KA (2017), Heat transfer in magnetohydrodynamic second grade fluid with porous impacts using Caputo-Fabrizio fractional derivatives, Journal of Mathematics, 49(2), pp.113-125.

[4] Abro KA, Hussain M \& Baig MM (2017), Slippage of magnetohydrodynamic fractionalized Oldroyd-B fluid in porous medium, Journal of Mathematics, 80(1), pp. 69-80.

[5] Sharma k \& Gupta S (2016), Analytical study of MHD boundary layer flow and heat transfer towards a porous exponentially stretching sheet in presence of thermal radiation, International Journal of Advances in Applied Mathematics and Mechanics, 4(1), pp. 1-10.

[6] Eldesoky IM, Kamel MH \& Abumandour RM (2014), Numerical study of slip effect of unsteady MHD pulsatile flow through porous medium in an artery using generalized differential quadrature method (Comparative Study), World Journal of Engineering and Technology, pp. 131-148.

[7] Ahmad I, Ilyas H \& Bilal M (2014), Numerical solution for nonlinear MHD Jeery-Hamel blood flow problem through neural networks optimized techniques, J. Appl. Environ. Biol. Sci., 4, pp. 33 43.

[8] Sedaghatizadeh N, Barari A, Soleimani S \& Modi M (2013), Analytical and numerical evaluation of steady flow of blood through artery, Biomedical Research, 24(1), pp. 88-98.

[9] Rathod VP \& Ravi M (2014), Blood flow through stenosed inclined tubes with periodic body acceleration in the presence of magnetic field and its applications to cardiovascular diseases, International Journal of Research in Engineering and Technology, pp. 96-101.

[10] Elshahed M (2000), MHD flow of an elastico-viscous fluid under periodic body acceleration, Int. J. Math. \& Math. Sci., 23(11), pp. 795-799.

[11] Ibraheem GH \& Abdulhadi AM (2014), Exact solutions for MHD flow of a viscoelastic fluid with the fractional Burgers' model in an annular pipe, International Journal Of Modern Engineering Research (IJMER), 4(3), pp. 58-64.

[12] Mohan V, Prasad V \& Varshney NK (2013), Effect of magnetic field on blood flow (elastico-viscous) under periodic body acceleration in porous medium, Journal of Mathematics, 6(4), pp. 43-48.

[13] Kumar D (2010), Application of Sumudu transform in the timefractional Navier-Stokes equation with MHD flow in porous media, Journal of Applied Sciences Research, 16(11), pp. 1814-1821.

[14] Liu Y \& Ma J (2015) Accelerating MHD flow of a generalized Oldroyd-B, Frontiers in Heat and Mass Transfer, 17, pp. 1-5. 